Acta Crystallographica Section D

\section{Biological Crystallography}

ISSN 0907-4449

a Department of Molecular, Cellular and Developmental Biology, Yale University, New Haven, CT 06511, USA, ' ${ }^{\text {The Northeastern }}$ Collaborative Access Team (NE-CAT), Advanced Photon Source, Argonne National Laboratory, Argonne, IL 60439, USA, 'Department of Chemistry, Yale University, New Haven, CT 06511, USA, and ${ }^{\mathbf{d}}$ Howard Hughes Medical Institute, Chevy Chase, MD 20815, USA

₹ Current address: Schrödinger Inc., New York, NY 10036, USA.

Correspondence e-mail: marco.marcia@yale.edu, anna.pyle@yale.edu

\title{
Solving nucleic acid structures by molecular replacement: examples from group II intron studies
}

Structured RNA molecules are key players in ensuring cellular viability. It is now emerging that, like proteins, the functions of many nucleic acids are dictated by their tertiary folds. At the same time, the number of known crystal structures of nucleic acids is also increasing rapidly. In this context, molecular replacement will become an increasingly useful technique for phasing nucleic acid crystallographic data in the near future. Here, strategies to select, create and refine molecularreplacement search models for nucleic acids are discussed. Using examples taken primarily from research on group II introns, it is shown that nucleic acids are amenable to different and potentially more flexible and sophisticated molecularreplacement searches than proteins. These observations specifically aim to encourage future crystallographic studies on the newly discovered repertoire of noncoding transcripts.

\section{Introduction}

The crystallographic phase problem (Muirhead \& Perutz, 1963) can be solved using different approaches, of which molecular replacement (MR) has been the most widely used since its development in the early 1960s (Hoppe, 1957; Rossmann \& Blow, 1962; Huber, 1965). Particularly today, MR can be broadly applied given that the number of structures, and thus of potential search models, is increasing exponentially in the open-access data banks (Rossmann, 2001). As of 19 February 2013, statistics run on the Protein Data Bank (PDB) show that of the almost 80000 deposited macromolecular X-ray structures, about $60 \%$ were solved by MR.

In addition to the abundance of search models, the popularity of MR is also a consequence of the flexibility of this method, which is applicable to solving the structures of any type of macromolecule ranging from proteins to nucleic acids and their complexes. However, proportionally more protein than nucleic acid X-ray structures have currently been solved by MR. Why is there a discrepancy between these two classes of macromolecules? Do these numbers indicate that nucleic acids are intrinsically less amenable to structure determination by MR? A number of considerations need to be made in regard to this issue.

Interestingly, the first protein and nucleic acid structures determined by MR were obtained at approximately the same time. Specifically, the structures of the Tobacco mosaic virus capsid protein (Jack, 1973) and of glyceraldehyde-3-phosphate dehydrogenase (Argos et al., 1975) were determined only shortly before the structure of tRNA ${ }^{\text {fMet }}$ (Woo et al., 1980). However, the rate of structure determination did not proceed at the same pace for the two classes of macromolecules. Today, nucleic acid X-ray structures represent only $1.8 \%$ of the total number of structures available in the PDB, and the structures
Received 23 February 2013

Accepted 14 May 2013 
Table 1

Different MR strategies for nucleic acids of different lengths.

\begin{tabular}{|c|c|c|c|}
\hline & Short & Medium & Long \\
\hline Size (nt) & $<30-40$ & $40-200$ & $>200$ \\
\hline Typical secondary/tertiary structure & Hairpins & Combinations of hairpins & Complex tertiary structures \\
\hline Availability of experimental models $\dagger(\%)$ & 83.5 & 13.1 & 3.4 \\
\hline Identification of structural homologues & $\begin{array}{l}\text { Based on size, independently } \\
\text { of sequence }\end{array}$ & $\begin{array}{l}\text { By structure-similarity algorithm } \\
\text { (sequence covariation) }\end{array}$ & $\begin{array}{l}\text { By structure-similarity algorithm } \\
\text { (sequence covariation) }\end{array}$ \\
\hline Strategies to improve MR success & Generally unnecessary & $\begin{array}{l}\text { Pruning bases/bases and sugars } \\
\text { Deletion of loops and junctions }\end{array}$ & $\begin{array}{l}\text { Pruning bases/bases and sugars } \\
\text { Using only selected domains } \\
\text { Supporting the MR search using } \\
\text { preliminary experimental phases }\end{array}$ \\
\hline Limitations of MR using experimental models & Internal helical symmetry & R.m.s.d. $\neq$ up to $2-3 \AA$ & R.m.s.d. $\neq$ up to $2-3 \AA$ \\
\hline $\begin{array}{l}\text { Reference models in the absence } \\
\text { of experimental data }\end{array}$ & Ideal helices modeled manually & $\begin{array}{l}\text { Combinations of ideal helices } \\
\text { modeled manually or } \\
\text { Three-dimensional motifs modeled } \\
\text { de novo or } \\
\text { Homology models }\end{array}$ & $\begin{array}{l}\text { Combinations of ideal helices } \\
\text { modeled manually (rare) or } \\
\text { Three-dimensional motifs modeled } \\
\text { de novo or } \\
\text { Homology models }\end{array}$ \\
\hline $\begin{array}{l}\text { Limitations of MR using in silico } \\
\text { designed models }\end{array}$ & Internal helical symmetry & $\begin{array}{l}\text { Difficulty in assigning small } \\
\text { helical domains } \\
\text { R.m.s.d. } \neq \text { up to } 1-1.5 \AA\end{array}$ & $\begin{array}{l}\text { Difficulty in assigning small } \\
\text { helical domains } \\
\text { R.m.s.d. } \ddagger \text { up to } 1-1.5 \AA\end{array}$ \\
\hline References & Baikalov \& Dickerson (1998) & Scott $(2012)$ & $\begin{array}{l}\text { Humphris-Narayanan \& Pyle (2012), } \\
\text { Marcia \& Pyle (2012) and this work }\end{array}$ \\
\hline
\end{tabular}

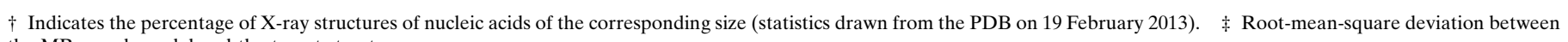
the MR search model and the target structure.

of protein-nucleic acid complexes make up $4.8 \%$ of the total. In contrast, protein-only structures account for the remaining 93.4\% (Fig. 1). With so few potential search models, it is not surprising that a smaller percentage of nucleic acid structures have been solved by MR.

Nucleic acid structure determination lags behind protein structure determination primarily because it has only recently been discovered that there are a wealth of structured noncoding RNA elements in cells (Washietl et al., 2007; The Encode Project Consortium, 2012; Fig. 1). Certainly, there is no theoretical or practical preclusion for the success of crystallographic studies on nucleic acids. In fact, the physics underlying crystallization, the geometry of the diffraction experiment and the choices of the methods used for structure

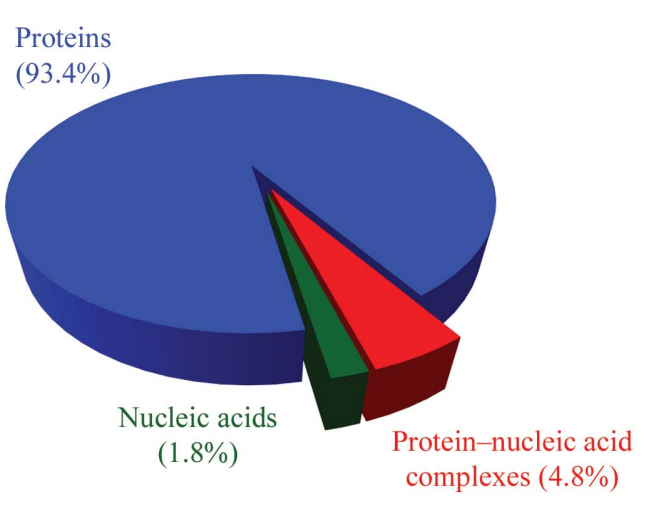

(a)

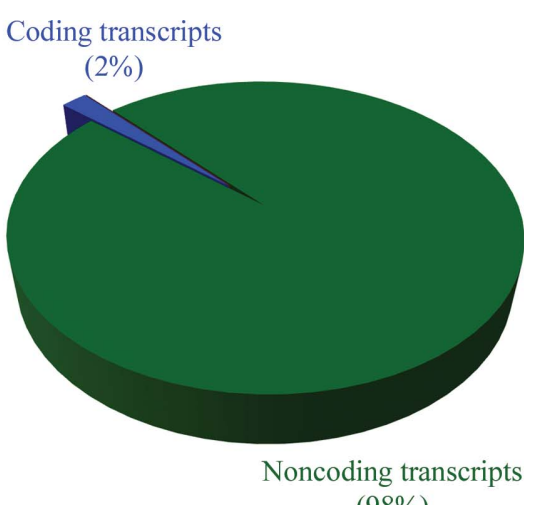

(b)
Figure 1

Statistics for nucleic acid versus protein structures. (a) Pie chart describing the percentage of X-ray structures solved containing different types of macromolecules. Nucleic acids are significantly underrepresented relative to proteins. Statistics were calculated from the PDB as of 19 February 2013. (b) Distribution of coding versus noncoding transcripts in the cell, as derived from genomic data analysis (Washietl et al., 2007; The Encode Project Consortium, 2012). An increasingly large number of noncoding elements have been shown to possess distinct tertiary structures; therefore, the quantity and the variety of nucleic acid crystallography targets are rapidly increasing. determination are essentially the same for both nucleic acids and proteins (Scott, 2012). Moreover, the vast majority of software currently used for data analysis and structure determination can process nucleic acid structures as efficiently as protein structures. However, in comparison to protein crystallography, nucleic acid crystallography does present certain technical and practical challenges (Baikalov \& Dickerson, 1998). These differences strongly affect the choice and the design of MR search models, which is the most crucial step in obtaining correct structure solutions (Evans \& McCoy, 2008). Therefore, in this report we discuss the preparation of search models for use in solving nucleic acid structures by MR. Specifically, we present examples and test cases related to our research on the structure of group II introns, which are 400-1000-nucleotide noncoding RNAs that are capable of self-splicing and retrotransposition (Pyle, 2010).

\subsection{Properties of nucleic acids}

Just like proteins, nucleic acids possess distinctive features at the primary, secondary and tertiary structure levels. These distinctions influence not only the function of these macromolecules but also their structural organization. Taking these properties into account is important when developing software to determine, analyze and model the three-dimensional structures of nucleic acids. Therefore, consideration of these differences is also critical when solving nucleic acid structures via MR. 
At the sequence level, nucleic acids are composed of simpler building blocks than proteins. Their sequence is less variegated, as it consists of a combination of only four nucleobases rather than the 20 amino acids that compose protein chains. As discussed below, this property makes nucleic acid structure less sensitive to differences in sequence, which is advantageous when applying the MR approach to nucleic acids.

Also, the secondary structure of nucleic acids, which refers to the helices typical of DNA and RNA (Moore, 1999), is simpler than that of proteins. DNA generally forms helical structures of the B-form (or, occasionally, the A-form or Z-form), while RNA molecules are primarily built from combinations of A-form helices (Scott, 2012). All of these motifs have a well known conserved geometry, so that (with some exceptions) their secondary structure can be predicted with a higher degree of confidence than protein secondary structure (Baikalov \& Dickerson, 1998). The high internal symmetry of nucleic acid helices adds nontrivial complications to the MR search functions (Baikalov \& Dickerson, 1998). However, in some cases, such symmetry may constitute an advantage for the MR approach by allowing structure solution even in the absence of experimental models (see below).

Despite the simplicity of their sequences and secondary structures, nucleic acids adopt intricate tertiary architectures and a wide variety of recurring three-dimensional motifs. The elaborate architecture of RNA is apparent from recent structures of ribosomes, riboswitches, self-splicing introns and many other RNAs (Butcher \& Pyle, 2011). The identification of RNA tertiary motifs is enriching our understanding of the nucleic acid structural space and it will allow the compilation of useful 'libraries' of nucleic acid structural building blocks. The latter will be useful in the future for identifying appropriate search models for MR.

Finally, the length of nucleic acid molecules is an important factor. In this report, we divide nucleic acids into three classes. Nucleic acids of up to 50 bases are defined as small, those of 50-200 nucleotides in length as medium and those with more than 200 nucleotides as long. Each of these three groups possesses properties that favor or hamper the MR approach to differing extents (Table 1).

\subsection{Choice and design of suitable MR models for nucleic acids}

As mentioned above, the most crucial step in solving structures of macromolecules by MR is the identification of a good search model. Such a model is characterized by high structural similarity to the target [i.e. a root-mean-square deviation (r.m.s.d.) of <2.5 ̊; Evans \& McCoy, 2008]. In most cases the structural similarity between the model and target cannot be determined accurately a priori, so MR typically requires a trial-and-error process (Evans \& McCoy, 2008). However, in general the coordinates of the model can be (i) derived from available experimental data, such as other X-ray or nuclear magnetic resonance (NMR) structures (Jung \& Zweckstetter, 2004; Langmead et al., 2004; Mao et al., 2011;
Thompson et al., 2012), or (ii) created in the absence of experimental data (Claude et al., 2004; Giorgetti et al., 2005; DiMaio et al., 2011). In the former case, the model can be represented (i) by a homologous structure, (ii) by the structure of the target molecule that is itself in a different state, space group or conformation or (iii) by the structures of domains of (homologues of) the target molecule. In the latter case, the model can instead be designed (iv) by homology modeling (Giorgetti et al., 2005) or (v) by de novo structure predictions (Thompson \& Baker, 2011). Recently, thanks to new software that randomly searches the accessible data banks, broader less user-biased trials can also be performed, thus enhancing the chances of obtaining interpretable MR solutions (Stokes-Rees \& Sliz, 2010). Finally, the input of weak experimental phases determined by anomalous scattering can enhance the chances of success of the MR search (Kleywegt \& Jones, 1997).

In the following paragraphs, we will describe these different strategies applied to nucleic acids and we will support our statements with examples from our own research and that of other investigators.

\section{MR searches in the presence of experimental models}

\subsection{Structural homology among nucleic acids}

In protein crystallography, a sequence identity of $30-35 \%$ to the target is generally accepted as a criterion for selecting MR search models (Chothia \& Lesk, 1986; Claude et al., 2004; DiMaio et al., 2011). This cutoff can be lowered further if the alignment between model and the target is highly accurate (Schwarzenbacher et al., 2004).

For nucleic acids, the sequence-to-structure correlations are less well understood than for proteins and current statistics suggest that they are weaker (Capriotti \& Marti-Renom, 2010). It has been proposed that short RNA loops need to share a sequence identity of $75 \%$ to infer structural similarity with confidence (Schudoma et al., 2010) and this increases to $85 \%$ in paralogous mRNA pairs (Chursov et al., 2012). At the same time, many noncoding RNA molecules possess well conserved secondary and tertiary folds but share low sequence similarity (Dirheimer et al., 1995; Zhang et al., 2005; Yu, 2011). Therefore, identifying suitable structural homologues for new nucleic acid targets may be challenging using only sequencesimilarity software, i.e. BLAST (Altschul et al., 1990), MUSCLE (Edgar, 2004), T-Coffee (Notredame et al., 2000) or ClustalW (Thompson et al., 1994).

This limitation may be overcome by considering the fact that nucleic acids possess greater evolutionary flexibility than proteins. Indeed, nucleic acid helices may possess identical structures even if their sequences differ dramatically, provided that the base pairing between the partner strands is maintained. Identifying evolutionary covariations in the sequences of partner strands is possible through comparative sequence analysis (Fox \& Woese, 1975; Pace et al., 1989; Woese \& Pace, 1993). This method can help to identify clusters of homologous molecules that do not share significant sequence conservation but possess common secondary-structure motifs and thus 
potentially similar tertiary structures. Therefore, structural similarity rather than sequence-similarity search algorithms need to be used to identify homologues of nucleic acids, i.e. CMfinder (Yao et al., 2006), Infernal (Nawrocki et al., 2009) or LocARNA (Will et al., 2007), or software performing wholegenome alignment ( $\mathrm{Yu}, 2011)$.

\subsection{MR searches on nucleic acids are not sensitive to sequence conservation}

Since nucleic acids may possess homologous structures even if they share very low sequence identity, we investigated the sensitivity of MR searches to nucleic acid sequence divergence using data sets and structures obtained in our laboratory for the Oceanobacillus iheyensis group II intron (Toor et al., 2008; Marcia \& Pyle, 2012). Briefly, group II introns are a broad class of self-splicing RNA molecules that are essential for gene expression in many organisms (Pyle \& Lambowitz, 2006). They constitute a good system both to exemplify sequence covariation in RNA and to study the sensitivity of MR programs to sequence divergence, mainly because all group II introns are expected to possess a common structural fold (Michel et al., 2009; Toor et al., 2009) despite the sequences of different group II intron subtypes having diverged significantly (Michel et al., 1989; Michel \& Costa, 1998).
For our investigation, we generated two random RNA sequences of 390 nucleotides in length (corresponding to the length of the crystallized form of the $O$. iheyensis group II intron). In one case, we used the Random DNA Sequence Generator software (http://www.faculty.ucr.edu/ mmaduro/ random.htm), obtaining a sequence with $21 \%$ identity to the $O$. iheyensis group II intron. In the other case, we artificially designed a completely new sequence with no identity to the $O$. iheyensis group II intron, mutating all purines to pyrimidines and vice versa, thus bringing the sequence divergence to an extreme case. We used these artificial sequences to modify the identity of the nucleobases in a structure of the $O$. iheyensis group II intron (PDB entry 3igi; Toor et al., 2010) using Coot (Emsley et al., 2010), thereby changing the primary structure but not the secondary and tertiary structures of 3igi. Finally, for each case we created a series of MR search models by exploring the dynamics of the RNA backbone in the range of r.m.s.d. values from 0 to $4 \AA$ using normal-mode analysis in FRODA (Fulle \& Gohlke, 2008). Phasing attempts in Phaser (McCoy, 2007) using the experimental structure-factor amplitudes of the structure with PDB code 4faw (Marcia \& Pyle, 2012) were successful with both sets of models, having r.m.s.d. values of up to $2-3 \AA$ from the original (Fig. 2). As expected, these results show that MR searches for nucleic acids are not affected by sequence divergence, only by the structural similarity between search model and target.

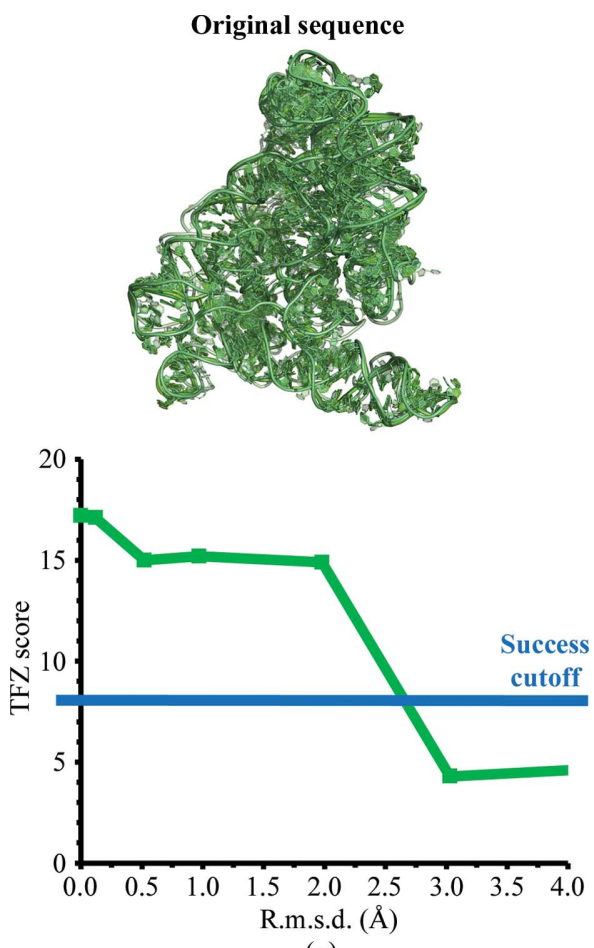

Figure 2
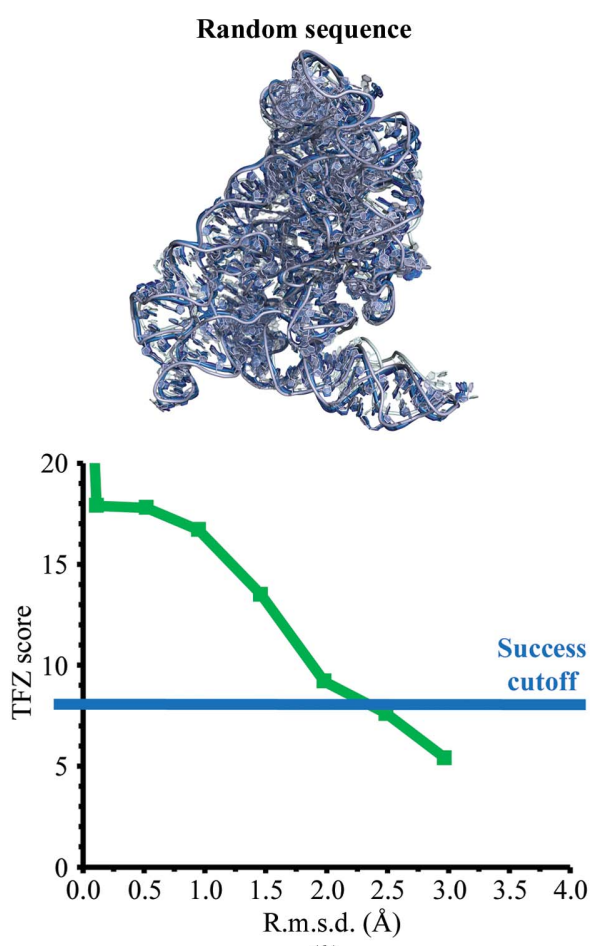

(b)
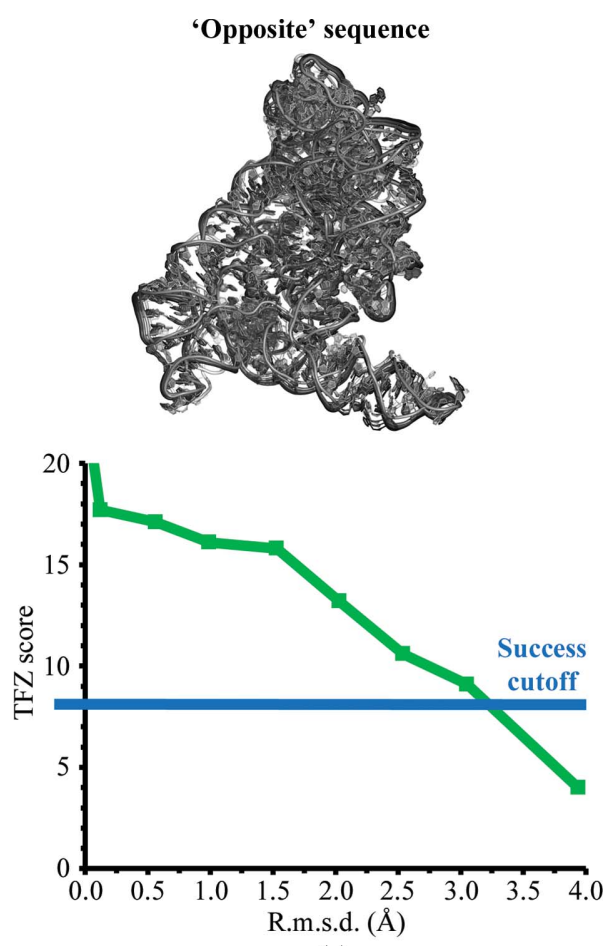

$(c)$

The low sensitivity of MR searches to nucleic acid sequence conservation. Group II intron structures were derived from the $O$. iheyensis group II intron structure (PDB entry 3igi) using $(a)$ the original intron sequence (green tones), $(b)$ a randomly generated sequence (blue tones) or (c) an 'opposite' sequence (gray tones). For each sequence, the backbone was distorted using $F R O D A$ with increasing values of the r.m.s.d. (from $0 \AA$ in the darkest color to $4 \AA$ in the lightest color). The TFZ scores from Phaser are plotted for each MR run. A TFZ value of 8 or higher was observed to confidently indicate correct solutions for protein structures (McCoy, 2007) and is thus taken here as a cutoff level for successful solutions. Although such a TFZ scale may not necessarily apply exactly to nucleic acid structures, we observed that our solutions with TFZ $>8$ were generally associated with interpretable electrondensity maps. 
Based on the considerations above, we conclude that structure-similarity searches accounting for sequence covariation are necessary to identify nucleic acid homologues and we suggest that the low level of sequence conservation of such homologues need not limit their use as search models for MR approaches.

\subsection{Minimal MR search models for nucleic acids}

Two factors explain the low sensitivity of MR programs to sequence divergence in nucleic acids, namely the high structural similarity of the four nucleobases and the fact that the nucleobases contribute only minimally to the total X-ray scattering. Rather than the identity of the nucleobases, the position of the sugar-phosphate backbone of nucleic acids is a much stronger determinant for the success of MR searches. Indeed, MR search models of nucleic acids can be pruned by removing all nucleobases, and in some cases also the sugar moieties, without affecting the success of the phasing process. To exemplify the latter concept, we again used the group II intron data as a test case. From a series of structures obtained with FRODA from PDB entry 3igi and characterized by increasing r.m.s.d. values $(0-4 \AA)$, we derived substructures removing either all of the nucleobase atoms, the nucleobase and sugar moieties or the sugar and phosphate groups. We then performed MR runs in Phaser, attempting to phase the experimental structure-factor amplitudes of PDB entry 4 faw. We determined that the sugar-phosphate backbone (with r.m.s.d. values of up to $2 \AA$ relative to the original structure) and even the phosphate groups by themselves (with r.m.s.d. values of up to $1 \AA$ ) are sufficiently good starting models to obtain correct solutions in Phaser, with TFZ scores greater than 8 and interpretable output electron-density maps. However, the bases alone do not possess sufficient scattering information for the MR software to phase the target data set correctly, regardless of the r.m.s.d. (Fig. 3).

These tests suggest that it is possible to use minimal MR models composed only of backbone elements. This is similar to what is performed in protein structure determination with the use of polyalanine (DeLano \& Brünger, 1995), polyglycine (Fabiane et al., 1998; Hausrath et al., 1999) or polyserine (Storici et al., 1999; Minor et al., 2000) models.

\subsection{Isomorphism of nucleic acid structures}

Another common case for MR is the study of targets whose structures are known but for which new data have been obtained, i.e. in novel crystallization conditions, space groups or functional states. For such situations, $100 \%$ sequence conservation is not a guarantee of structural identity (Kosloff \& Kolodny, 2008) and MR may still be unsuccessful if the structural similarity is not maintained. Indeed, strong structural differences of $>6 \AA$ r.m.s.d. between pairs of identical targets can be caused by the binding of ligands or cofactors, by the influence of solvent molecules, by the adoption of alternative conformations, by the establishment of alternative quaternary-structure interactions or by domain swapping (Liu \& Eisenberg, 2002; Kosloff \& Kolodny, 2008).

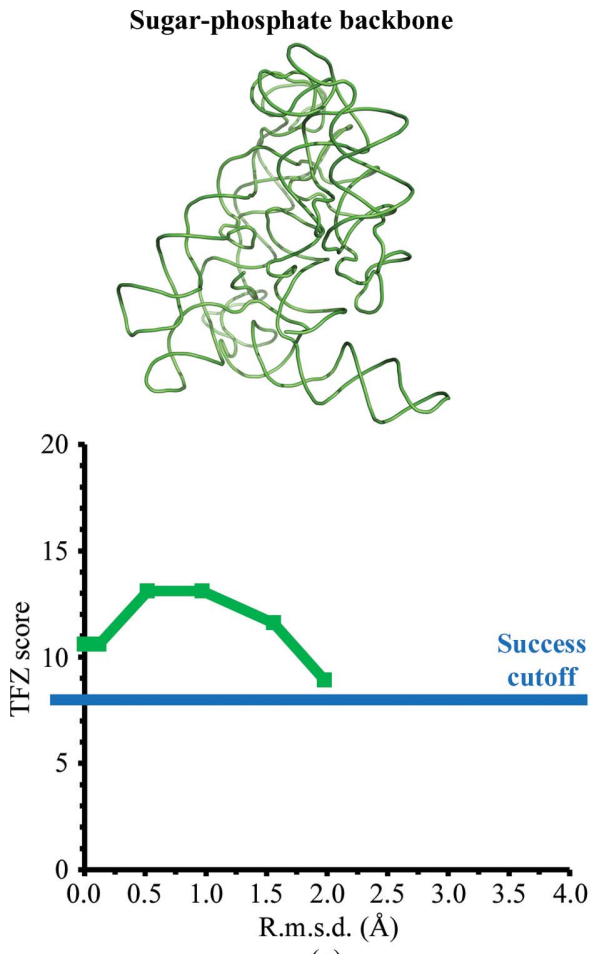

Figure 3
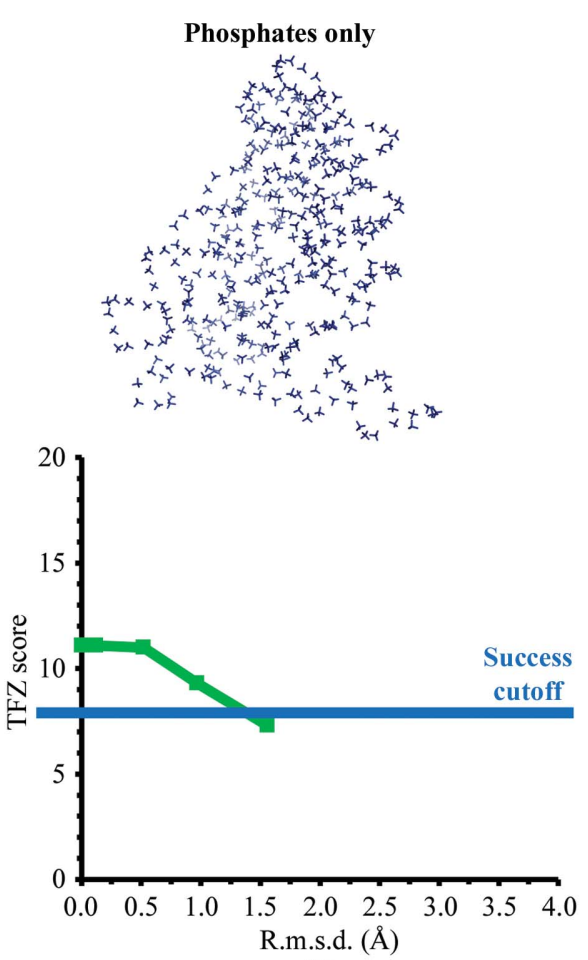

(b)
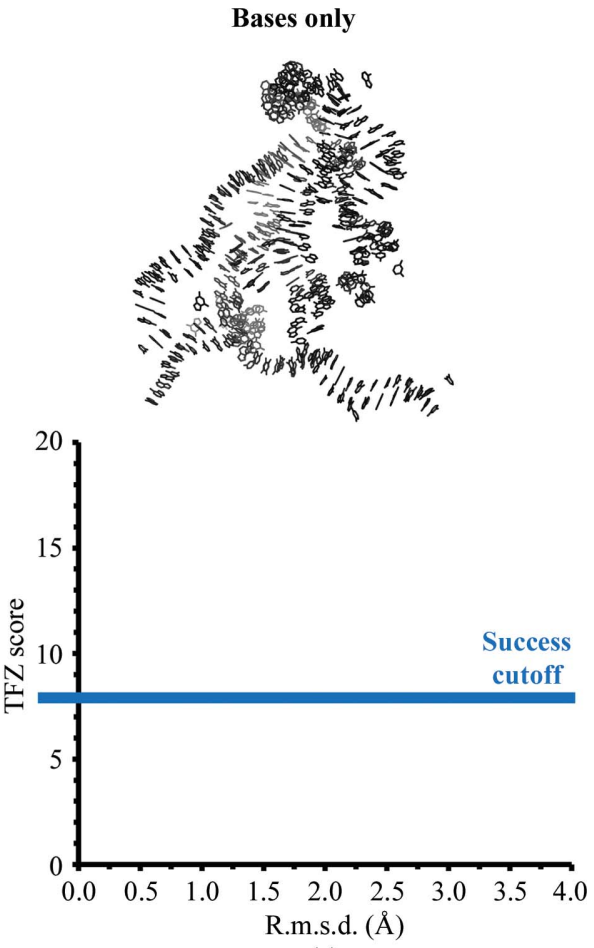

(c)

Minimal MR models for nucleic acid structures. The structure of the group II intron can be successfully solved (TFZ $>8$ in Phaser and interpretable electron-density maps) using only the sugar-phosphate backbone distorted by up to $2 \AA$ r.m.s.d. (a) or only the phosphate groups distorted by up to $1 \AA$ r.m.s.d. (b) but not by using only the coordinates of the nucleobases (no Phaser solution was obtained) $(c)$. 
Moreover, certain amino-acid sequences adopt different secondary structures depending on their molecular environment, as exemplified by moonlighting proteins (Jeffery, 1999) and promiscuous enzymes (Khersonsky et al., 2006). The same considerations are believed to affect nucleic acids considerably more frequently than proteins. For instance, the structure of group II intron domain 5 (D5) in isolation is significantly different from that in the context of the intact enzyme (Zhang \& Doudna, 2002; Sigel et al., 2004; Toor et al., 2008). Such behavior can be attributable to two main reasons. Firstly, the free-energy landscape of nucleic acid molecules is flatter than that of proteins, implying that a given nucleic acid sequence may possess a large number of favored conformations (Zuker, 1989). It has been estimated that RNA molecules of $N$ nucleotides can fold into about $1.8^{N}$ based-paired secondary structures with many potential tertiary folds (Zuker \& Sankoff, 1984; Hajdin et al., 2010). Secondly, nucleic acid structures may be more dependent on solvent molecules and ions than proteins. For example, metals determine and stabilize the structure of certain nucleic acid motifs (Butcher \& Pyle, 2011), such as the kink turn (Goody et al., 2004), and they also modulate nucleic acid flexibility (Al-Hashimi et al., 2003). As further evidence of the importance of metal ions in nucleic acids, the three-dimensional X-ray structures of these macromolecules contain an average of approximately 15 metal ligands (Stefan et al., 2006; Schnabl et al., 2012), while metalloproteins typically contain only 1-5 (Nakamura et al., 2009). As a result, small changes in solvent molecules can cause very pronounced non-isomorphism in nucleic acid structures. This non-isomorphism can lead to difficulties in MR and similarly affects heavy-atom replacement techniques such as single/multiple isomorphous replacement (SIR/MIR; Baikalov \& Dickerson, 1998).

However, we recently reported a rather surprising result showing that a nucleic acid molecule can be far more accommodating to different solvent conditions than is generally expected. During our work on the $O$. iheyensis group II intron, we were able to crystallize our target with a wide variety of metal-ion combinations, including alkali metals $\left(\mathrm{Li}^{+}\right.$, $\mathrm{Na}^{+}, \mathrm{K}^{+}, \mathrm{Rb}^{+}$and $\left.\mathrm{Cs}^{+}\right)$, alkaline-earth metals $\left(\mathrm{Mg}^{2+}\right.$ and $\left.\mathrm{Ba}^{2+}\right)$ and post-transition metals $\left(\mathrm{Tl}^{+}\right)$, and with non-metallic ions $\left(\mathrm{NH}_{4}^{+}\right)$(Marcia \& Pyle, 2012). While other research groups used transition metals in combination with physiological ions (i.e. $\mathrm{K}^{+}$and $\mathrm{Mg}^{2+}$ ) to characterize their targets (Stahley et al., 2007), with the group II intron we were successful in obtaining well diffracting crystals when we fully replaced the physiological metals $\mathrm{K}^{+}$and $\mathrm{Mg}^{2+}$ with analogues directly in the crystallization buffer. The resulting crystal structures revealed a surprising degree of adaptation in accommodating all of these ions (Marcia \& Pyle, 2012). We obtained a total of 14 different structures, all isomorphous to each other (r.m.s.d. of $\sim 1 \AA$ ), and we could efficiently solve all of them using MR. These structures allowed us to detect conformational changes in the intron and to visualize different intermediate catalytic forms of this ribozyme, which allowed an improved understanding of the catalytic mechanism (Marcia \& Pyle, 2012). Additionally, the identification of these new features confirms that our structures did not suffer from model bias, which is a matter of great concern during MR experiments, especially when using models that are very similar to the targets (Kleywegt, 2000; Terwilliger, 2004). In summary, our work shows that large noncoding RNAs can indeed maintain the same fold in the presence of nonphysiological ions. This allows metal soaks and MR to serve as a valuable tool for determining and interpreting the structural and functional properties of nucleic acids.

\subsection{Multi-domain MR searches for nucleic acids}

In the previous sections, we described the use of the entire $O$. iheyensis group II intron structure as a single search model in MR searches. However, typical MR pipelines do not account for rigid-body motions amongst the different domains that compose three-dimensional structures. Therefore, these pipelines may fail if the individual domains are not used as separated search ensembles (Cygler \& Anderson, 1988a,b). For multi-domain proteins, important factors for the success of MR are the completeness of the composite model, the size and number of the individual domains and, in some cases, the order in which the domains are used in the iterative MR search (Evans \& McCoy, 2008; Luo et al., 2011).

Here, we discuss some facts to consider when generating domain ensembles for nucleic acids. The group II intron can provide an illustrative example, as it is composed of six domains (Pyle, 2010). Briefly, domain 1 (D1; 265 nucleotides long in $O$. iheyensis) is the most intricate and is composed of six smaller subdomains (D1i-ii, D1a, D1b, D1c, D1d1 and D1d2). Each subdomain primarily consists of a relatively simple and short helical fragment (Pyle, 2010). The other domains protrude out from the D1 structural core and are smaller in size. In the $O$. iheyensis group II intron construct engineered in our laboratory for crystallization purposes (Toor et al., 2008), these other domains possess the following characteristics. Domain 2 (D2) is formed by a single eightbase-pair stem terminating in a tetraloop (20 nucleotides in total). Domains 3 and 4 (D3 and D4) both contain two stems separated by a bulged region, with D3 longer than D4 (38 and 27 nucleotides, respectively). D5 is 34 nucleotides long and has a characteristic 'elbow' shape (Pyle, 2010). The tertiary structure of D6 is still undetermined (Pyle, 2010).

We considered two strategies to create individual domain ensembles for the group II intron. We first followed a strategy that is typical in protein crystallography. We used ensembles covering each of the five domains from a previously published intron structure (PDB entry 3igi; Toor et al., 2008). We implemented this strategy using Phaser (McCoy, 2007) and a more recent data set (PDB entry 4faw; Marcia \& Pyle, 2012). The MR search was quick and it converged to a solution characterized by high scores for the three larger domains $(\mathrm{TFZ}=57.8$ for $\mathrm{D} 1, \mathrm{TFZ}=33.9$ for D3 and TFZ $=42.8$ for D5). These results provided an interpretable electron-density map suitable for further structural refinement. Only the two smaller domains of the intron (D2 and D4) were placed incorrectly in the unit cell. This test suggests that when the 
structures of individual domains are known and are maintained in the context of the entire molecule it is possible to perform nucleic acid multi-domain searches successfully, similar to the searches performed for protein structures.

Our second approach to solving the group II intron structure via MR used individual short helical fragments as independent ensembles. We first removed junction nucleotides from the search structure (PDB entry 3igi) and then divided the structure into ten helical fragments, each representing a single subdomain, including each separate subdomain of D1. Together, these helices covered approximately $85 \%$ of the intron structure. The helices varied in length, but many were structurally similar, with reciprocal r.m.s.d.s of approximately $4 \AA$ or less. Thus, we expected an MR search to be complex and unsuccessful. However, Phaser was able to model eight of the ten helical fragments into the structure, resulting in a high score $(\mathrm{TFZ}=17.7)$ and an interpretable electron-density map (Fig. 4). Interestingly, successful solutions were also obtained when the backbone of the individual subdomains used in the search were distorted by normal-mode analysis in FROD $A$ by up to $1 \AA$ r.m.s.d. with respect to their original structure in PDB entry 3igi (Fig. 4).

These results are encouraging because they suggest that small helical fragments can be a powerful tool for solving large nucleic acid structures, rather than confining the technique to small and medium-sized molecules (Robertson et al., 2010). Given our results above, we currently believe that a maximum of 6-8 helical fragments, each not shorter than 12-15 base pairs, could be used as MR search models without making the MR search too long or too complex. These numbers may increase if more elaborate models possessing distinctive folds are designed, i.e. models containing bulges, loops and junctions. These considerations point towards the potential of exploiting de novo modeling tools that can build a variety of fragments for use as MR search models (HumphrisNarayanan \& Pyle, 2012; see below).

\section{Phased-MR approaches}

In our multi-domain structural search using the ten intron subdomains, we noticed that Phaser encountered problems in placing two domains (D2 and D4). These domains possess less distinct tertiary motifs and are the shortest domains in the structure. To aid in the assignment of such small domains, information from weak experimental phases could potentially support and enhance the MR solution (Kleywegt \& Jones, 1997). Therefore, we attempted to identify the correct positions of D2 and D4 using phased MR. This analysis involved
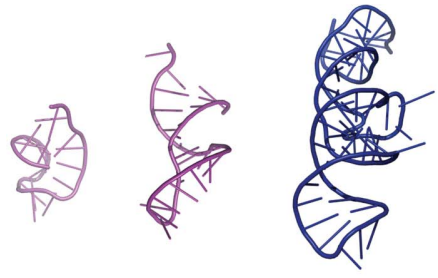

D1a D1b

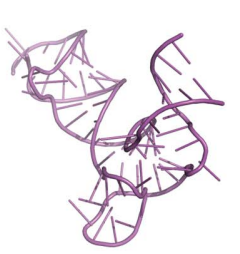

D1d1

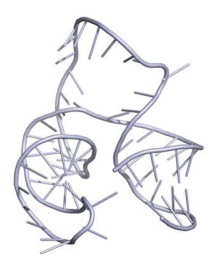

D1d2

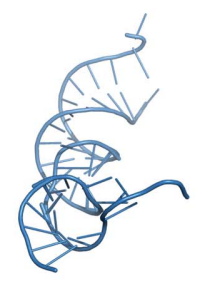

D1i-ii

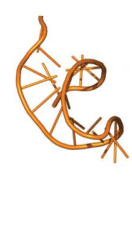

D2

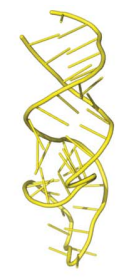

D3

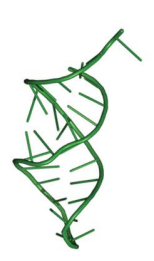

D4

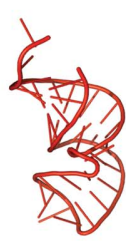

D5

(a)

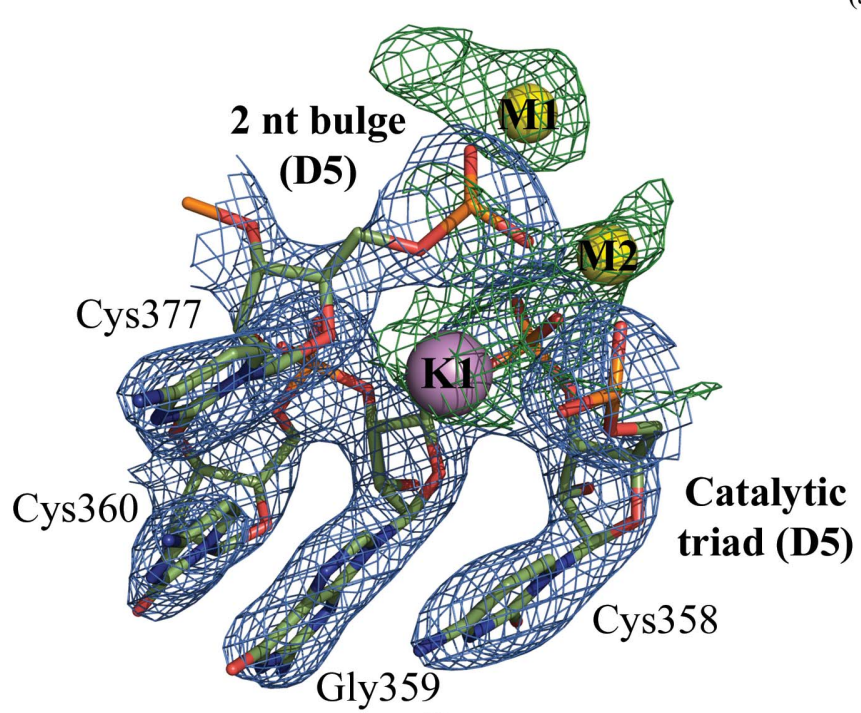

(b)

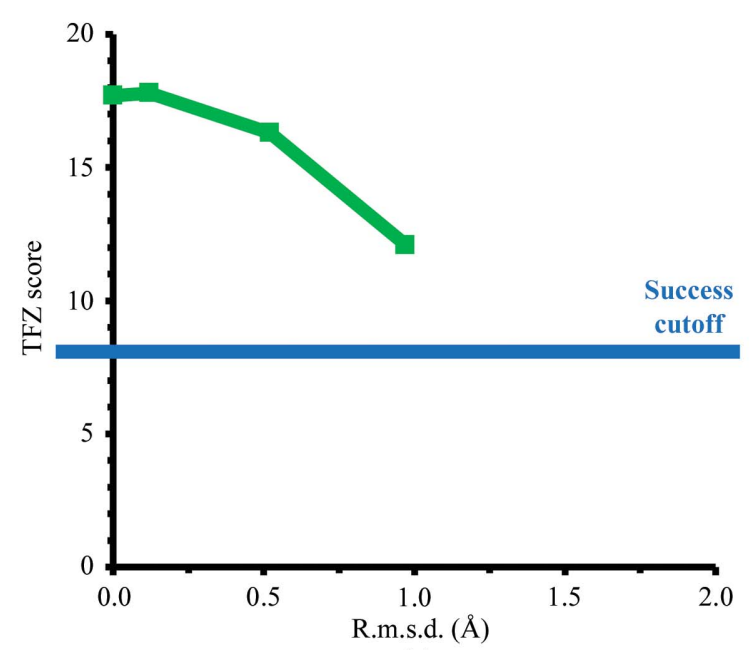

(c)

Figure 4

Multi-domain MR searches for phasing nucleic acid structures. (a) The experimental structure-factor amplitudes of an $O$. iheyensis group II intron data set (PDB entry 4faw) can be phased with a Phaser multi-domain MR search using ten individual intron subdomains as a starting model. (b) The resulting $\sigma$-weighted $2 F_{\mathrm{o}}-F_{\mathrm{c}}$ electron-density map is shown around the active-site motifs in blue mesh $(1.5 \sigma$ contour level). The positive signal in the $\sigma$-weighted $F_{\mathrm{o}}-F_{\mathrm{c}}$ map (green mesh, $3.0 \sigma$ ) is shown at the expected position of the catalytic metal ions, which were not included in the search model (M1/M2 and K1; yellow and purple spheres, respectively). (c) Successful solutions (TFZ $>8$ ) could be obtained using intron subdomain structures distorted by up to $1 \AA$ r.m.s.d. with respect to their original structure in the model (PDB entry 3igi). 
the structures of D2 and D4 extracted from 3igi along with poor experimental phases obtained from a data set for the group II intron cocrystallized with $\mathrm{Yb}^{3+}$ (Toor et al., 2008). The phase information provided by this single data set was insufficient to produce a traceable electron-density map (Toor $e t$ al., 2008), but it enabled us to place both D2 and D4 correctly using MOLREP (Vagin \& Teplyakov, 2010). This process was also successful when using D2 and D4 structures that had been distorted by up to $1 \AA$ r.m.s.d. in FRODA (Fig. 5). Notably, we obtained successful MOLREP solutions by excluding higher resolution shells from the calculation, possibly because the phase information for these shells is of lower accuracy than the low-resolution and intermediate-resolution shells.

The phased-MR approach can also be run iteratively, where it places the different domains of the intron into the asymmetric unit one after another. This potentially enhances the probability of identifying the position of smaller domains. However, this strategy does not significantly help the difficult process of placing domains whose structures differ greatly from the target molecule. For instance, attempts to position D2 or D4 models with greater than 1 A r.m.s.d. distortion were unsuccessful even after positioning D1, D3 and D5.

Thus, experimental phases and MR can be used synergistically. Heavy-atom soaking can be used successfully in nucleic acid crystallography (see \$2.4) but it often yields experimental phases of poor quality (Toor et al., 2008). Combining this information with MR searches significantly increases the success of structure solution.

\section{MR searches in the absence of experimental models}

Traditionally, fragments and domains from previously published structures are used to create MR search models. However, polypeptide fragments created using in silico design

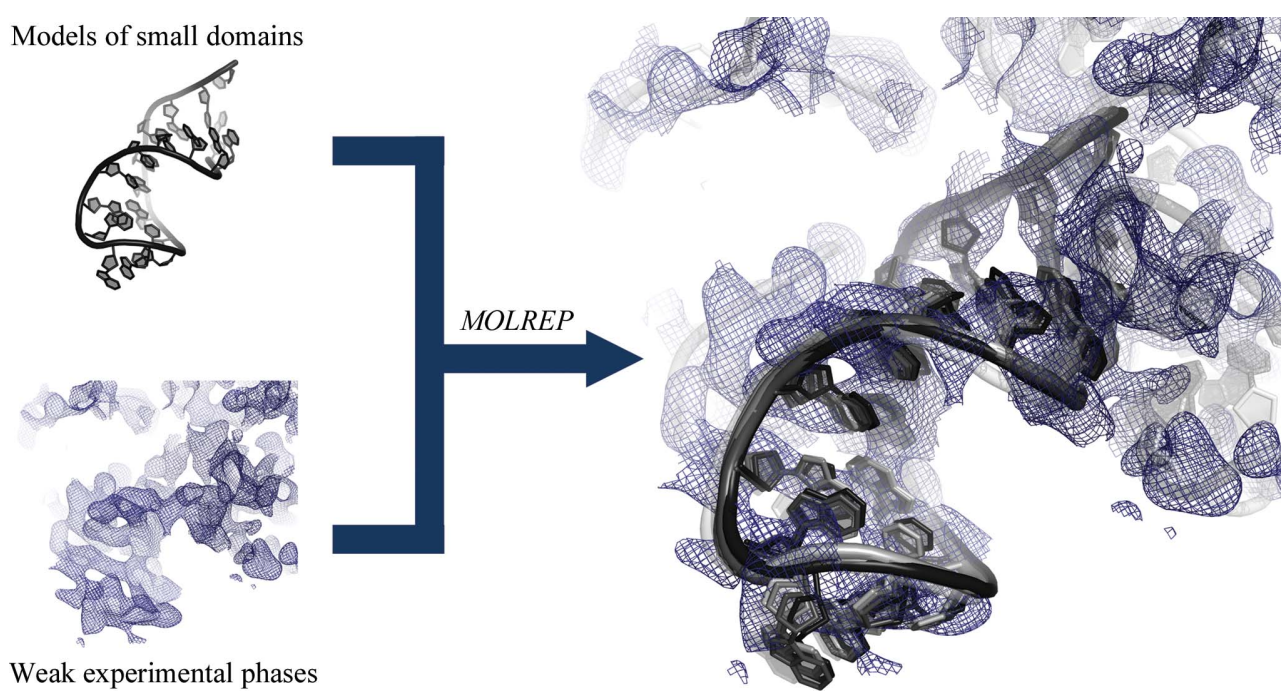

Figure 5

Phased MR. The D2 domain of the group II intron (black cartoon diagram; top left panel) was not placed correctly in multi-domain MR searches (see Fig. 4). However, it can be assigned to its expected position in the electron density (right panel) by MOLREP if information from weak experimental phases is provided (bottom left panel). The solvent-flattened experimental electron-density map is depicted as a blue mesh at a $1.5 \sigma$ contour level. are becoming increasingly common MR search models thanks to the ever-growing richness of the structural databases, the availability of computational power and the improved accuracy of prediction algorithms (Gubbi et al., 2007). Similarly, polynucleotide structures computed in silico can also be used as search models for MR of nucleic acids. To date, idealized helical fragments have been used for the study of short DNA chains (Baikalov \& Dickerson, 1998) and of RNA molecules of medium size, such as the 70-nucleotide L1-ligase ribozyme (Robertson \& Scott, 2007; Robertson et al., 2010; Scott, 2012). While in certain cases helices with ideal geometry may also be used in the study of large nucleic acids, structure solution of the latter significantly benefits from the use of more sophisticated search models, i.e. models generated by homology modeling or de novo design.

\subsection{Homology modeling of nucleic acid structures}

Homology modeling is the most successful structureprediction method for proteins and it is routinely used to guide various experimental studies (Martí-Renom et al., 2000; Lukk et al., 2012). Thanks to the efforts of CASP (Critical Assessment of Structure Prediction), fully automated methods are available for modeling proteins with respectable accuracy (Kryshtafovych et al., 2011; Mariani et al., 2011). In addition, there are databases of three-dimensional models of proteins for which the structure of at least one homolog is known and deposited in the PDB (Pieper et al., 2004). However, very few methods are available for performing homology modeling on nucleic acids (Rother, Rother, Boniecki et al., 2011). This is largely owing to the difficulty in identifying structural homologs. As mentioned above, there are far fewer nucleic acid structures available than there are for proteins, and nucleic acid homology is difficult to detect using only sequence data. Methods that incorporate secondary-structure data, such as FASTR3D (Lai et al., 2009), RNAFRABASE (Popenda et al., 2008) and FR3D (Sarver et al., 2008), are more successful in identifying nucleic acid templates. Furthermore, an accurate alignment between the target and the template is also critical for homology modeling. Rather than sequence-based alignments, secondary-structure-based alignment methods such as $R$-Coffee (Moretti et al., 2008) offer greater accuracy. Additionally, manual adjustment is frequently necessary to improve the quality of the alignment beyond what is currently possible using automated methods (Rother, Rother, Puton et al., 2011). Once the template has been identified and 
aligned, tools such as ModeRNA (Rother, Rother, Puton et al., 2011) or RNAbuilder (Flores \& Altman, 2010; Flores et al., 2010) allow the construction of a three-dimensional model.

\subsection{De novo design of nucleic acid structural models}

In addition to models constructed using homology, de novo modeling can also be used to aid MR in a number of ways. Small helices, tertiary motifs or potentially entire domains can be generated de novo and used as search fragments. Recently, an energy-based modeling protocol (FARFAR) was shown to model over half of 32 RNA motifs to within 1-2 $\AA$ r.m.s.d. accuracy (Das et al., 2010). Thus, de novo generation of tertiary components for MR may currently be feasible. Further, in cases where a suitable homology model exists, de novo modeling can be used to refine regions that contain insertions or deletions. For example, exhaustively generating and evaluating RNA loop conformations can lead to accurate de novo models for small RNA loops (Sripakdeevong et al., 2011).

An additional and important use for modeling is to generate hundreds or even thousands of structural models de novo or from a starting homology model or backbone trace. 'Core' regions of a starting model that were highly conserved within a multiple sequence alignment could be modeled consistently among each ensemble member. Simultaneously, a de novo sampling or optimization protocol could be used to generate greater conformational variability in other regions. Strategies for introducing conformational variability include the sampling of alternative structures with an r.m.s.d. close to a starting structure (Humphris-Narayanan \& Pyle, 2012) or the use of an energy-based sampling protocol such as FARFAR (Das et al., 2010). A subset of low-energy de novo models can then be selected to solve the phase problem using MR. If MR using homology models or tertiary components has already generated density that is insufficient for all-atom autobuilding, this initial density could be utilized as an additional energy term to help select low-energy models. Once de novo models have been selected for MR, a new set of phases could be calculated to generate an improved electron-density map. Ensemble modeling and density-based refinement of homology models has been highly successful for MR in proteins (DiMaio et al., 2011). Thus, a similar strategy of de novo model optimization and refinement may aid in solving RNA structures using MR in the future (Fig. 6).

\section{Optimizing and refining MR models for nucleic acids with RCrane}

Once a model has been chosen, its optimization and manual refinement may be beneficial and may increase the chances of success of the MR search. RCrane is a methodology for constructing and correcting RNA backbone structure (Keating \& Pyle, 2010, 2012) and allows such model optimizations. This technique uses both the RNA pseudotorsions (Wadley et al., 2007) and the RNA backbone conformers (Richardson et al., 2008) to accurately predict and build allatom RNA structures starting from only the phosphate and base positions. RCrane uses the $\theta^{\prime}$ and $\eta^{\prime}$ pseudotorsions, where $\theta^{\prime}$ is defined as the $\mathrm{P}_{i}-\mathrm{C}_{i}^{\prime}-\mathrm{P}_{i+1}-\mathrm{C1}_{i-1}^{\prime}$ dihedral and $\eta^{\prime}$ is defined as the $\mathrm{Cl}_{i-1}^{\prime}-\mathrm{P}_{i}-\mathrm{Cl}_{i}^{\prime}-\mathrm{P}_{i+1}$ dihedral (Keating \& Pyle, 2010). These pseudotorsions allow an accurate characterization of the RNA backbone using a coarse-grained representation. Conversely, the RNA backbone conformers enumerate roughly 50 detailed backbone configurations using the standard backbone torsions $\delta_{-1}, \varepsilon_{-1}, \zeta_{-1}, \alpha, \beta, \gamma$ and $\delta$

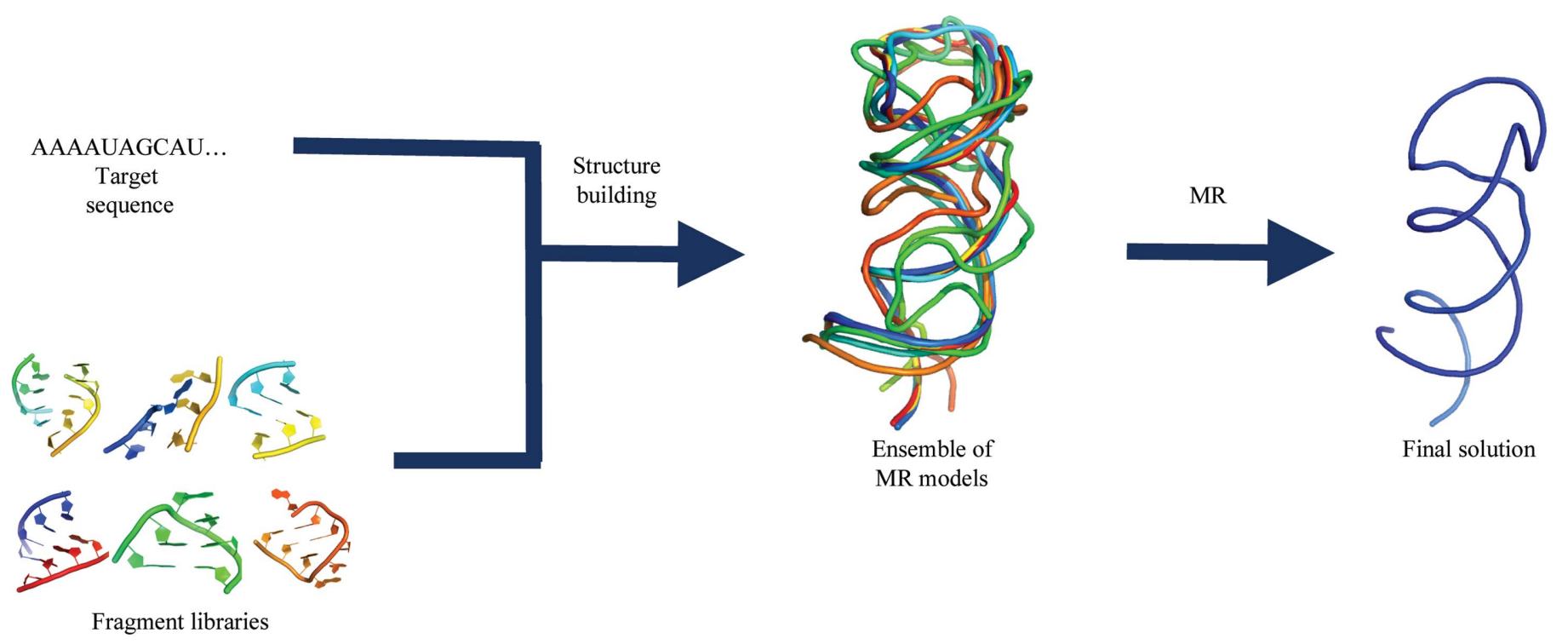

Figure 6

De novo design of nucleic acid domains to be used as MR search ensembles. Using a target sequence and a fragment library (left-hand panel), de novo design techniques can be used to build hundreds or thousands of models (middle panel). Each model could be scored based on an energy function and, if applicable, its accuracy of fit to an initial experimental density. Promising models could then be used as starting points for further tracing and refinement. Iterative model rebuilding and energy-guided optimization could significantly increase the quality of the final solution (right-hand panel, blue structure). 
(Richardson et al., 2008). RCrane uses the pseudotorsions and related coarse-grained information to predict the appropriate backbone conformer with high accuracy: one of the first three conformer predictions is correct $98 \%$ of the time and the first prediction is correct $84 \%$ of the time. Additionally, this technique is highly tolerant of errors in the phosphate and $\mathrm{Cl}^{\prime}$ coordinates. Errors of up to $1 \AA$ in the phosphate coordinate lead to only a $6 \%$ decrease in prediction accuracy, and errors of up to $2 \AA$ in the $\mathrm{Cl}^{\prime}$ coordinate lead to only a 13\% decrease in prediction accuracy (Keating \& Pyle, 2010). After conformer prediction, a multidimensional minimization procedure is used to build the remaining backbone coordinates.

RCrane is implemented as a plugin for Coot (Emsley et al., 2010) and is frequently used for building RNA into lowresolution electron density solved through traditional phasing approaches such as SAD or MAD. However, the technique is equally applicable to structures solved using MR. As discussed above, search models may be constructed using homology modeling. The techniques used for homology modeling frequently build structures that match the overall architecture of the RNA, but the detailed backbone structure contains numerous steric clashes and improper backbone configurations. These issues can lead to improper placement of phosphates, which are particularly important in MR owing to their scattering power. Because of the high error tolerance of the RCrane predictions, the technique is still able to accurately determine the appropriate backbone conformer despite these improper phosphate placements. The minimization procedure used for building the backbone conformer can then also be used to correct the placement of the phosphate atoms, even in the absence of an electron-density map. These corrections allow RCrane to notably improve the phasing power of the MR search model.

RCrane is also applicable after the structure has been phased. In RCrane 1.1 or newer, the extend-chain option can be used to build missing portions of the model, as it allows the crystallographer to build onto the end of an existing RNA segment. This feature is particularly useful when phasing via $M R$, as the replacement model frequently does not cover all of the crystallized nucleotides. Additionally, RCrane can be used to correct portions of the model that do not match the newly phased electron density. Nucleotides that need minor adjustments can be corrected via rotamerization, which uses the existing phosphate and base positions to rebuild the backbone structure. Regions of the structure that need more dramatic corrections can be rebuilt from scratch using the extend-chain option. These correction procedures can continue to be used after crystallographic refinement to further improve the model (Keating \& Pyle, 2010, 2012).

\section{Conclusions and future perspectives}

In this report, we have examined structure determination using MR and highlighted the differences in this technique when solving nucleic acid rather than protein structures, with a particular emphasis on the selection and design of the search model. These steps are generally the most crucial in determining the success of MR approaches (Evans \& McCoy, 2008) and we envisage that they will become particularly important in the future for MR of nucleic acids. While the number of available nucleic acid structures does not yet match the number of protein structures, nucleic acid crystallography is rapidly expanding. Highly structured nucleic acids are now known to be essential players in an extensive variety of biological processes. Additionally, $98 \%$ of the transcriptome consists of noncoding transcripts (The Encode Project Consortium, 2007), and a large fraction of these are expected to adopt well defined stable tertiary structures in order to perform their cellular functions (Cruz \& Westhof, 2009; Novikova et al., 2012; Westhof \& Romby, 2010). These discoveries serve to further emphasize the importance of nucleic acid structure determination. Moreover, given their intrinsic properties, nucleic acids are also good targets for structure prediction de novo or by homology modeling. New computational approaches and software are being developed to create reliable three-dimensional models for medium-tolarge nucleic acids even in the absence of experimental data. These models can help to expand the size of the nucleic acid structural databases and to increase the use of MR. Owing to this and the other factors discussed above, we expect that the rate of nucleic acid structures solved by MR in the future will be comparable to, if not higher than, that of protein structures.

We acknowledge the beamline scientists at 24-ID-C and 24-ID-E, NE-CAT, APS, Argonne, Illinois, USA, where all of the diffraction data for the group II intron were collected. We thank all members of the Pyle laboratory for constructive discussion and critical reading of the manuscript. This project was supported by the National Institutes of Health (RO1GM50313). AMP is a Howard Hughes Medical Institute Investigator. KRR is supported by a grant from the National Institute of General Medical Sciences (8 P41 GM103403-10) of the National Institutes of Health. Use of the Advanced Photon Source was supported by the US Department of Energy, Office of Science, Office of Basic Energy Sciences under Contract No. W-31-109-Eng-38. EHN is supported by a grant from the National Institute of General Medical Sciences (F32GM096516) of the National Institutes of Health.

\section{References}

Al-Hashimi, H. M., Pitt, S. W., Majumdar, A., Xu, W. \& Patel, D. J. (2003). J. Mol. Biol. 329, 867-873.

Altschul, S. F., Gish, W., Miller, W., Myers, E. W. \& Lipman, D. J. (1990). J. Mol. Biol. 215, 403-410.

Argos, P., Ford, G. C. \& Rossmann, M. G. (1975). Acta Cryst. A31, 499-506.

Baikalov, I. \& Dickerson, R. E. (1998). Acta Cryst. D54, 324-333.

Butcher, S. E. \& Pyle, A. M. (2011). Acc. Chem. Res. 44, 1302-1311.

Capriotti, E. \& Marti-Renom, M. A. (2010). BMC Bioinformatics, 11, 322.

Chothia, C. \& Lesk, A. M. (1986). EMBO J. 5, 823-826.

Chursov, A., Walter, M. C., Schmidt, T., Mironov, A., Shneider, A. \& Frishman, D. (2012). Nucleic Acids Res. 40, 956-962. 
Claude, J.-B., Suhre, K., Notredame, C., Claverie, J.-M. \& Abergel, C. (2004). Nucleic Acids Res. 32, W606-W609.

Cruz, J. A. \& Westhof, E, (2009). Cell, 136, 604-609

Cygler, M. \& Anderson, W. F. (1988a). Acta Cryst. A44, 38-45.

Cygler, M. \& Anderson, W. F. (1988b). Acta Cryst. A44, 300-309.

Das, R., Karanicolas, J. \& Baker, D. (2010). Nature Methods, 7, 291-294.

DeLano, W. L. \& Brünger, A. T. (1995). Acta Cryst. D51, 740-748.

DiMaio, F., Terwilliger, T. C., Read, R. J., Wlodawer, A., Oberdorfer, G., Wagner, U., Valkov, E., Alon, A., Fass, D., Axelrod, H. L., Das, D., Vorobiev, S. M., Iwaï, H., Pokkuluri, P. R. \& Baker, D. (2011). Nature (London), 473, 540-543.

Dirheimer, G., Keith, G., Dumas, P. \& Westhof, E. (1995). $t R N A$ : Structure, Biosynthesis, and Function, edited by D. Söll \& U. L. RajBhandary, pp. 93-126. Washington: American Society for Microbiology.

Edgar, R. C. (2004). BMC Bioinformatics, 5, 113.

Emsley, P., Lohkamp, B., Scott, W. G. \& Cowtan, K. (2010). Acta Cryst. D66, 486-501.

Evans, P. \& McCoy, A. (2008). Acta Cryst. D64, 1-10.

Fabiane, S. M., Sohi, M. K., Wan, T., Payne, D. J., Bateson, J. H., Mitchell, T. \& Sutton, B. J. (1998). Biochemistry, 37, 12404-12411.

Flores, S. C. \& Altman, R. B. (2010). RNA, 16, 1769-1778.

Flores, S. C., Wan, Y., Russell, R. \& Altman, R. B. (2010). Pac. Symp. Biocomput., pp. 216-227.

Fox, G. E. \& Woese, C. R. (1975). Nature (London), 256, 505-507.

Fulle, S. \& Gohlke, H. (2008). Biophys. J. 94, 4202-4219.

Giorgetti, A., Raimondo, D., Miele, A. E. \& Tramontano, A. (2005). Bioinformatics, 21, ii72-ii76.

Goody, T. A., Melcher, S. E., Norman, D. G. \& Lilley, D. M. (2004). $R N A, \mathbf{1 0}, 254-264$.

Gubbi, J., Parker, M. \& Palaniswami, M. (2007). Applications of Fuzzy Sets Theory, edited by F. Masulli, S. Mitra \& G. Pasi, pp. 627634. Berlin/Heidelberg: Springer.

Hajdin, C. E., Ding, F., Dokholyan, N. V. \& Weeks, K. M. (2010). RNA, 16, 1340-1349.

Hausrath, A. C., Grüber, G., Matthews, B. W. \& Capaldi, R. A. (1999). Proc. Natl Acad. Sci. USA, 96, 13697-13702.

Hoppe, W. (1957). Acta Cryst. 10, 750-751.

Huber, R. (1965). Acta Cryst. 19, 353-356.

Humphris-Narayanan, E. \& Pyle, A. M. (2012). J. Mol. Biol. 421, $6-26$.

Jack, A. (1973). Acta Cryst. A29, 545-554.

Jeffery, C. J. (1999). Trends Biochem. Sci. 24, 8-11.

Jung, Y.-S. \& Zweckstetter, M. (2004). J. Biomol. NMR, 30, 11-23.

Keating, K. S. \& Pyle, A. M. (2010). Proc. Natl Acad. Sci. USA, 107, 8177-8182.

Keating, K. S. \& Pyle, A. M. (2012). Acta Cryst. D68, 985-995.

Khersonsky, O., Roodveldt, C. \& Tawfik, D. S. (2006). Curr. Opin. Chem. Biol. 10, 498-508.

Kleywegt, G. J. (2000). Acta Cryst. D56, 249-265.

Kleywegt, G. J. \& Jones, T. A. (1997). Acta Cryst. D53, 179-185.

Kosloff, M. \& Kolodny, R. (2008). Proteins, 71, 891-902.

Kryshtafovych, A., Fidelis, K. \& Moult, J. (2011). Proteins, 79, Suppl. 10, 196-207.

Lai, C.-E., Tsai, M.-Y., Liu, Y.-C., Wang, C.-W., Chen, K.-T. \& Lu, C. L. (2009). Nucleic Acids Res. 37, W287-W295.

Langmead, C. J., Yan, A., Lilien, R., Wang, L. \& Donald, B. R. (2004). J. Comput. Biol. 11, 277-298.

Liu, Y. \& Eisenberg, D. (2002). Protein Sci. 11, 1285-1299.

Lukk, T. et al. (2012). Proc. Natl Acad. Sci. USA, 109, 4122-4127.

Luo, D., Ding, S. C., Vela, A., Kohlway, A., Lindenbach, B. D. \& Pyle, A. M. (2011). Cell, 147, 409-422.

Mao, B., Guan, R. \& Montelione, G. T. (2011). Structure, 19, 757766.

Marcia, M. \& Pyle, A. M. (2012). Cell, 151, 497-507.

Mariani, V., Kiefer, F., Schmidt, T., Haas, J. \& Schwede, T. (2011). Proteins, 79, Suppl. 10, 37-58.
Martí-Renom, M. A., Stuart, A. C., Fiser, A., Sánchez, R., Melo, F. \& Sali, A. (2000). Annu. Rev. Biophys. Biomol. Struct. 29, 291325.

McCoy, A. J. (2007). Acta Cryst. D63, 32-41.

Michel, F. \& Costa, M. (1998). RNA Structure and Function, edited by R. W. Simons \& M. Grunberg-Manago, pp. 175-202. Cold Spring Harbor Laboratory Press.

Michel, F., Costa, M. \& Westhof, E. (2009). Trends Biochem. Sci. 34, 189-199.

Michel, F., Umesono, K. \& Ozeki, H. (1989). Gene, 82, 5-30.

Minor, D. L., Lin, Y.-F., Mobley, B. C., Avelar, A., Jan, Y. N., Jan, L. Y. \& Berger, J. M. (2000). Cell, 102, 657-670.

Moore, P. B. (1999). The RNA World, 2nd ed., edited by R. F. Gesteland, T. R. Cech \& J. F. Atkins. Cold Spring Harbor Laboratory Press.

Moretti, S., Wilm, A., Higgins, D. G., Xenarios, I. \& Notredame, C. (2008). Nucleic Acids Res. 36, W10-W13.

Muirhead, H. \& Perutz, M. F. (1963). Nature (London), 199, 633-638.

Nakamura, K., Hirai, A., Altaf-Ul-Amin, M. \& Takahashi, H. (2009). Plant Biotechnol. 26, 517-521.

Nawrocki, E. P., Kolbe, D. L. \& Eddy, S. R. (2009). Bioinformatics, 25, 1335-1337.

Notredame, C., Higgins, D. G. \& Heringa, J. (2000). J. Mol. Biol. 302, 205-217.

Novikova, I. V., Hennelly, S. P. \& Sanbonmatsu, K. Y. (2012). Bioarchitecture, 2, 189-199.

Pace, N. R., Smith, D. K., Olsen, G. J. \& James, B. D. (1989). Gene, 82, 65-75.

Pieper, U., Eswar, N., Braberg, H., Madhusudhan, M. S., Davis, F. P., Stuart, A. C., Mirkovic, N., Rossi, A., Marti-Renom, M. A., Fiser, A., Webb, B., Greenblatt, D., Huang, C. C., Ferrin, T. E. \& Sali, A. (2004). Nucleic Acids Res. 32, D217-D222.

Popenda, M., Blazewicz, M., Szachniuk, M. \& Adamiak, R. W. (2008). Nucleic Acids Res. 36, D386-D391.

Pyle, A. M. (2010). Crit. Rev. Biochem. Mol. Biol. 45, 215-232.

Pyle, A. M. \& Lambowitz, A. M. (2006). The RNA World, 3rd ed., edited by R. F. Gesteland, T. R. Cech \& J. F. Atkins, pp. 469505. Cold Spring Harbor Laboratory Press.

Richardson, J. S. et al. (2008). RNA, 14, 465-481.

Robertson, M. P., Chi, Y.-I. \& Scott, W. G. (2010). Methods, 52, 168-172.

Robertson, M. P. \& Scott, W. G. (2007). Science, 315, 1549-1553.

Rossmann, M. G. (2001). Acta Cryst. D57, 1360-1366.

Rossmann, M. G. \& Blow, D. M. (1962). Acta Cryst. 15, 24-31.

Rother, K., Rother, M., Boniecki, M., Puton, T. \& Bujnicki, J. M. (2011). J. Mol. Model. 17, 2325-2336.

Rother, M., Rother, K., Puton, T. \& Bujnicki, J. M. (2011). Nucleic Acids Res. 39, 4007-4022.

Sarver, M., Zirbel, C. L., Stombaugh, J., Mokdad, A. \& Leontis, N. B. (2008). J. Math. Biol. 56, 215-252.

Schnabl, J., Suter, P. \& Sigel, R. K. (2012). Nucleic Acids Res. 40, D434-D438.

Schudoma, C., May, P., Nikiforova, V. \& Walther, D. (2010). Nucleic Acids Res. 38, 970-980.

Schwarzenbacher, R., Godzik, A., Grzechnik, S. K. \& Jaroszewski, L. (2004). Acta Cryst. D60, 1229-1236.

Scott, W. G. (2012). Acta Cryst. D68, 441-445.

Sigel, R. K., Sashital, D. G., Abramovitz, D. L., Palmer, A. G., Butcher, S. E. \& Pyle, A. M. (2004). Nature Struct. Mol. Biol. 11, 187-192.

Sripakdeevong, P., Kladwang, W. \& Das, R. (2011). Proc. Natl Acad. Sci. USA, 108, 20573-20578.

Stahley, M. R., Adams, P. L., Wang, J. \& Strobel, S. A. (2007). J. Mol. Biol. 372, 89-102.

Stefan, L. R., Zhang, R., Levitan, A. G., Hendrix, D. K., Brenner, S. E. \& Holbrook, S. R. (2006). Nucleic Acids Res. 34, D131-D134.

Stokes-Rees, I. \& Sliz, P. (2010). Proc. Natl Acad. Sci. USA, 107, 21476-21481. 
Storici, P., Capitani, G., De Biase, D., Moser, M., John, R. A., Jansonius, J. N. \& Schirmer, T. (1999). Biochemistry, 38, 8628-8634. Terwilliger, T. C. (2004). Acta Cryst. D60, 2144-2149.

The Encode Project Consortium (2007). Nature (London), 447, 799-816.

The Encode Project Consortium (2012). Nature (London), 489, $57-74$.

Thompson, J. \& Baker, D. (2011). Proteins, 79, 2380-2388.

Thompson, J. D., Higgins, D. G. \& Gibson, T. J. (1994). Nucleic Acids Res. 22, 4673-4680.

Thompson, J. M., Sgourakis, N. G., Liu, G., Rossi, P., Tang, Y., Mills, J. L., Szyperski, T., Montelione, G. T. \& Baker, D. (2012). Proc. Natl Acad. Sci. USA, 109, 9875-9880.

Toor, N., Keating, K. S., Fedorova, O., Rajashankar, K., Wang, J. \& Pyle, A. M. (2010). RNA, 16, 57-69.

Toor, N., Keating, K. S. \& Pyle, A. M. (2009). Curr. Opin. Struct. Biol. 19, 260-266.

Toor, N., Keating, K. S., Taylor, S. D. \& Pyle, A. M. (2008). Science, 320, $77-82$.

Vagin, A. \& Teplyakov, A. (2010). Acta Cryst. D66, 22-25.
Wadley, L. M., Keating, K. S., Duarte, C. M. \& Pyle, A. M. (2007). J. Mol. Biol. 372, 942-957.

Washietl, S. et al. (2007). Genome Res. 17, 852-864.

Westhof, E. \& Romby, P. (2010). Nature Methods, 7, 965-967.

Will, S., Reiche, K., Hofacker, I. L., Stadler, P. F. \& Backofen, R. (2007). PLoS Comput. Biol. 3, e65.

Woese, C. R. \& Pace, N. R. (1993). The RNA World, 1st ed., edited by R. F. Gesteland \& J. F. Atkins, pp. 91-117. Cold Spring Harbor Laboratory Press.

Woo, N. H., Roe, B. A. \& Rich, A. (1980). Nature (London), 286, 346-351.

Yao, Z., Weinberg, Z. \& Ruzzo, W. L. (2006). Bioinformatics, 22, 445-452.

Yu, M. K. (2011). MEng thesis. Massachusetts Institute of Technology, Boston, Massachusetts, USA.

Zhang, L. \& Doudna, J. A. (2002). Science, 295, 2084-2088.

Zhang, S., Haas, B., Eskin, E. \& Bafna, V. (2005). IEEE/ACM Trans. Comput. Biol. Bioinform. 2, 366-379.

Zuker, M. (1989). Science, 244, 48-52.

Zuker, M. \& Sankoff, D. (1984). Bull. Math. Biol. 46, 591-621. 